\title{
Stellungnahme der Leitenden Ärztinnen und Ärzte am UniversitätsSpital Zürich zu einem DRG-Moratorium
}

Zum Thema dieses Beitrags siehe auch die FMH-Erklärung auf Seite 924
Sehr geehrter Herr Bundesrat Burkhalter

Wir Leitenden Ärztinnen und Ärzte am UniversitätsSpital Zürich (USZ) wenden uns gegen die Einführung der DRG-Fallpauschalen zum 1.1.2012 und sprechen uns für ein DRG-Moratorium aus.

Hierfür gibt es eine Reihe von schwerwiegenden und stichhaltigen Gründen:

- Die Hauptargumente für die Einführung der DRGs, nämlich Kosteneinsparungen und Qualitätsverbesserung, werden inzwischen nicht einmal mehr von der Geschäftsführung der SwissDRG AG aufrecht erhalten. Es wird sogar von dieser Seite - die hoffentlich zuverlässig Bescheid weiss - von einer initialen Kostensteigerung gesprochen. Diese Kostensteigerung ist vorwiegend durch die notwendige Neuschaffung von Stellen im Bereich der Verwaltung und die entsprechenden Hard- und Softwareanschaffungen bedingt. Sie kommt nicht den Patienten zugute.

- Die als Besonderheit der Schweiz immer wieder hervorgehobene Begleitforschung zur DRG-Einführung findet bisher nicht statt. Damit ist das Hauptargument für einen Schweizer Sonderweg und eine kontrollierte Einführung der DRGs in der Schweiz nicht mehr gegeben. Die Begleitforschung müsste mindestens ein Jahr vor Beginn der DRGs einsetzen, um sinnvoll zu sein. Auswirkungen lassen sich nur feststellen, wenn die Ausgangsbedingungen bekannt sind. Dies ist bisher nicht der Fall. Damit entfällt ein zentrales Argument für eine Einführung der DRGs zum 1.1.2012.

- Die ärztliche Weiterbildung ist bisher konzeptionell im DRG-System überhaupt nicht berücksichtigt und verankert. Sie kann nicht - wie aktuell geplant - über individuelle Verhandlungen der einzelnen Spitäler mit den Kassen gewährleistet werden, da es für die ärztliche Weiterbildung schweizweit ein einheitliches Konzept und einheitliche Richtlinien gibt. Diese einheitlichen Richtlinien sind nicht durch individuelle Lösungen ersetz- oder austauschbar.

- Durch die DRGs wird ein erhöhter Verwaltungsaufwand notwendig, der ärztliche Ressourcen bindet und damit die Zeit am und für den Patien- ten weiter limitiert. Der Verwaltungsaufwand wird steigen, die Zeit, die für die Patienten aufgewendet werden kann, wird auf pflegerischer und ärztlicher Seite sinken. Das ist für eine gute Patientenversorgung kontraproduktiv.

- Die Schweiz wird durch die DRGs einen Verlust von Innovationsmöglichkeiten in der Medizin erleben. Innovationen werden sich nur verspätet in Fallpauschalen abbilden lassen. Insbesondere innovative Spitäler wie die Universitätsspitäler werden einen Verlust an Qualität und innovativer Spitzenmedizin zu verzeichnen haben. Sie können somit ihrer eigentlichen Aufgabe, der Weiterentwicklung der Medizin, nicht mehr in vollem Umfang gerecht werden.

- Die DRGs verzerren die Bedingungen zugunsten von privaten Anbietern. Dem durch Gesetze entgegenzuwirken, wird z.B. in Zürich bereits versucht. Gleichzeitig legen die DRGs jedoch Festpreise für Leistungen unterschiedlicher Qualität fest. Dies entspricht einer sozialistischen Planwirtschaft und keiner Wettbewerbssteigerung.

- Die einzigen übrigbleibenden Argumente, die für die Einführung der DRGs sprechen, sind diejenigen der Transparenz und der Vergleichbarkeit von medizinischen Leistungen. Brötchen, die gleich viel kosten, schmecken aber noch lange nicht gleich gut. Eine einheitliche Preisfestsetzung sagt nichts über die Qualität der Leistungen aus.

Wir Leitenden Ärztinnen und Ärzte am UniversitätsSpital Zürich fordern daher eine Rückkehr zur Vernunft und Besinnung. Statt des Beharrens auf offensichtlich unbrauchbaren Entscheidungen sollten effiziente, leistungsgerechte, transparente und sinnvolle Lösungen gesucht werden. Es sollten Lösungen gesucht werden, die nicht den Verwaltungsaufwand und die Bürokratie steigern, sondern den Patienten und damit Steuer- und Krankenkassenbeitragszahlern zugute kommen. Es wurden hier Entscheidungen über die Betroffenen - nämlich die Patienten - hinweg getroffen, die im höchsten Grad undemokratisch sind. Mit einem Moratorium zur Einführung der DRGs fordern wir daher auch die Festlegung einer Obergrenze für die Verwaltungskosten. Der Einfluss von Bürokraten 
auf medizinische Entscheidungen, auf Behandlungsnotwendigkeiten und sinnvolle Therapien muss begrenzt werden. Ethische Überlegungen müssen im Vordergrund stehen, nicht monetäre Interessen und Gewinnmaximierung. Das ethisch verantwortbare Patientenwohl ist jedoch bei der Planung der DRGs offensichtlich in den Hintergrund getreten.

Wir Leitenden Ärztinnen und Ärzte am UniversitätsSpital Zürich fordern ausserdem vor einer Einführung der DRGs die Klärung der Frage, wie die ärztliche Weiterbildung berücksichtigt und verankert werden kann und die Klärung der Stellung der Universitäts-

Mit freundlichen Grüssen

PD Dr. David Holzmann, Pfaffhausen

Prof. Dr. Dominik Straumann, Zürich

Prof. Dr. Jean-Claude Fauchère, Gockhausen

PD Dr. Gabriela Studer, Uerikon

Prof. Dr. Philippe Gertsch, Bäch

Prof. Dr. Jürg Hafner, Zürich

Dr. Roman Kocian, Pfaffhausen

PD Dr. Urs Schanz, Dübendorf

Prof. Dr. Hans Jung, Zürich

PD Dr. Stefan Hegemann, Zürich

Prof. Dr. Huldrych Günthard, Zürich

Prof. Dr. Reinhard Dummer, Zürich

Dr. Walter Künzi, Zürich

Prof. Dr. Thomas Pfammatter, Zürich

Prof. Dr. Beat Müllhaupt, Ennetbaden

Prof. Dr. Roger Lehmann, Zürich

PD Dr. Christoph Thalhammer, Hersberg

PD Dr. Oliver Distler, Zürich

PD Dr. Stephan Segerer, Illnau

Dr. Pius Brühlmann, Küsnacht

PD Dr. Urs Schwarz, Küsnacht

PD Dr. Daniel Schmid, Zollikon

PD Dr. Dominique Bettex, Zürich

Prof. Dr. Barbara Buddeberg, Zürich

PD Dr. Marc Husmann, Zürich

Prof. Dr. Peter Schmid, Oberwil spitäler mit ihren Innovationsmöglichkeiten für die Medizin. Ein Vorbote der Entwicklung ist sicher die avisierte Einsparung von 48 Millionen Franken am UniversitätsSpital Zürich. Wir fordern eine Klärung der Fragestellungen für die Begleitforschung und eine Etablierung einer solchen Forschung. Zudem fordern wir, dass auch öffentlich eingeräumt wird, dass DRGs nicht zu einer Kosteneinsparung führen werden. Wir sprechen uns also im Sinne der eigentlichen Aufgabe eines Gesundheitssystems, nämlich der Heilung von Kranken, für ein DRG-Moratorium aus.

PD Dr. Michael Müntener, Zürich

Prof. Dr. Dr. Jens Funk, Zürich

Prof. Dr. Georg Noll, Zürich

Prof. Dr. Paul Schneider, Zürich

Prof. Dr. Barbara Ballmer-Weber, Winterthur

Prof. Dr. Alexander Huber, Küsnacht

PD Dr. Nicolas Müller, Zürich

Prof. Dr. Dr. Gerhard Rogler, Zürich

Prof. Dr. Thomas Fehr, Zürich

Dr. Didier Schneiter, Zürich

PD Dr. Emanuela Keller, Kilchberg

PD Dr. Nicole Ochsenbein-Kölble, Zürich

Dr. Peter Steiger, Gossau/ZH

Dr. Christoph Nöthiger, Zürich

Prof. Dr. Johann Steurer, Zürich

Prof. Dr. Peter Bauerfeind, Erlenbach

Prof. Dr. Christoph Schmid, Uster

PD Dr. Marco Zalunardo, Uster

PD Dr. Romaine Arlettaz, Feldmeilen

Prof. Dr. Rosmarie Caduff, Luzern

Prof. Dr. Mario Lachat, Hinteregg

Prof. Dr. Spyridon Kollias, Erlenbach

Prof. Dr. Christian Ruef, Andelfingen

Prof. Dr. Annette Böhler, Zürich

Dr. Stephan Regenass, Basel 